\title{
An overview of FSHD diagnostic methods
}

\author{
N.V. Zernov ${ }^{1 *}$, M.Yu. Skoblov ${ }^{1,2,3}$ \\ ${ }^{1}$ Research Centre for Medical Genetics, Moscow, Russia \\ ${ }^{2}$ Moscow Institute of Physics and Technology, Dolgoprudny, Russia \\ ${ }^{3}$ Far Eastern Federal University, Vladivostok, Russia \\ *e-mail:nzernov01@gnmail.com
}

Key words: FSHD diagnostic, D4Z4 array, Southern blotting, molecular combing; nanopore sequencing, genotyping, methylation profiling

Motivation and Aim: Facial-scapular-humeral myodystrophy Landouzy-Dejerine (FSHD) is an autosomal dominant myodystrophy. The prevalence of the disease in different populations varies from 1: 20000 to $1: 14,000$ [1-4], conceding only to Duchenne muscular dystrophy and myotonic dystrophy. The basis of FSHD pathogenesis is ectopic expression of the transcription factor DUX4 in skeletal muscle encoded by D4Z4 repetitive units of subtelomeric region of chromosome 4q35 [5]. The D4Z4 repeats are widely presented in human genome and have high similarity between different chromosomes. This fact makes diagnostic of FSHD highly complicated for basic molecular diagnostic laboratories. In this paper, we review existing methods for FSHD molecular diagnostic thus conduce to choose the most suitable diagnostic technique in a basic molecular genetic laboratory.

Methods: Here we present a comprehensive analysis of current and possible methods for FSHD diagnostic: Southern blotting [6]; molecular combing [7]; nanopore sequencing [8]; STR haplotyping [9]; 4qA haplotyping [10]; methylation profiling [11]. Results: The most informative of currently existing methods is demonstrated by molecular combing; however, a price-informative ratio is the best for nonradioactive Southern blotting.

Conclusion: Based on the criteria of specificity, sensitivity, informative and price for today, the most suitable method for FSHD diagnostic in basic molecular genetic laboratory is the nonradioactive Southern blotting. This method is allow to diagnose about $90 \%$ of all FSHD cases, however it couldn't diagnose cases with low mosaicism, complex rearrangements between $4 \mathrm{qA} / 10 \mathrm{qA}$ allels. These issues resolve by the molecular combing method, but because of price of reagents and special equipment this method not suitable for basic molecular genetic diagnostic laboratory. The nanopore sequencing now have high price, not enough reads length and quality for FSHD diagnostic. However, the nanopore sequencing technology may be method of choice for the diagnostic in future because it has potential to resolve all limitations of currently existing methods.

\footnotetext{
References:

1. Padberg G.W. et al. (1995) Muscle Nerve Suppl. (2):S81-4.

2. Flanigan K.M. et al. (2001) Neuromuscul Disord. 11(6-7):525-9.

3. Mostacciuolo M.L. et al. (2009) Clin Genet. 75(6):550-5.

4. Norwood F.L. et al. (2009) Brain. 132(11):3175-86.

5. Snider L. et al. (2010) PLoS Genet. 6(10):e1001181.

6. Stefan J. (2007) White and Stuart Cantsilieris (eds.). Genotyping: Methods and Protocols. Methods in Mol Biol. 1492:107-125. DOI 10.1007/978-1-4939-6442-0 7.

7. Nguyen K., Walrafen P. et al. (2011) Ann Neurol. 70:627-6333.

8. Mitsuhashi S., Nakagawa S. (2017) Sci Rep. 7:14789.

9. Barat-Houari M. et al. (2010) Eur J Hum Genet. 18(5):533-538.

10. Papanikos F., Skoulatou C. et al. (2014) Clin Chim Acta. 429:96-103. DOI 10.1016/j.cca.2013.11.032.

11. Jones T., Yan C. et al. (2014) Clin Epigenetics. 6(1):23. DOI 10.1186/1868-7083-6-23.eCollection 2014.
} 